\title{
Further evidence that FSH causes bone loss independently of low estrogen
}

\author{
Jameel Iqbal · Harry C. Blair · Alberta Zallone • \\ Li Sun $\cdot$ Mone Zaidi
}

Received: 15 December 2011/ Accepted: 29 January 2012/Published online: 21 February 2012

(C) Springer Science+Business Media, LLC 2012

\section{Introduction}

The premise that menopause causes osteoporosis was first proposed by Fuller Albright at the Massachusetts General Hospital [1]. His hypothesis linking the loss of sex steroids to bone loss led to estrogen hormone replacement therapy (HRT) becoming the first successful treatment for osteoporosis [2]. More recently, however, a finer re-examination of menopausal bone loss has revealed that nearly half of lifetime loss occurs within the first 5 years [3, 4]. Bone loss accelerates dramatically during the late peri-menopause, and can, in fact, be most rapid up to 3 years before the last menstrual period [3-5] (Fig. 1a). During this early phase up to 5 years before the last menstrual period, estrogen levels are relatively unperturbed, whereas follicle-stimulating hormone (FSH) levels are rising to compensate for failing ovaries [6,7] (Fig. 1b). These most rapid rates of bone loss cannot conceivably be explained by low estrogen; other pathophysiological mechanisms must play a dominant role.

In the early 2000s, we showed that pituitary hormones can directly affect the skeleton by bypassing their usual endocrine targets. Thyroid-stimulating hormone (TSH) was first documented to regulate bone remodeling directly by

J. Iqbal $\cdot$ L. Sun $\cdot$ M. Zaidi $(\bowtie)$

The Mount Sinai Bone Program, Mount Sinai School of Medicine, New York, USA

e-mail: mone.zaidi@mssm.edu

H. C. Blair

Departments of Pathology and Cell Biology, University of Pittsburgh, and the VA Medical Center, Pittsburgh, USA

A. Zallone

Department of Anatomy and Histology, University of Bari, Bari, Italy acting on both osteoclasts and osteoblasts [8]. Not only did this study attribute the osteoporosis of hyperthyroidism, in part, to low TSH levels, it set forth a new paradigm for the existence of a pituitary-bone axis (Fig. 2). As part of this paradigm shift, we later discovered that $\mathrm{FSH}$ directly stimulates bone resorption [9]; thus the idea that rising FSH levels during the late peri-menopause could potentially contribute to the bone loss traditionally attributed solely to low estrogen [10]. We are by no means limiting the proven importance of low estrogen in causing bone loss. Instead, we suggest circumstances, such as the late peri-menopause, where FSH may play a dominant role.

Several studies have confirmed direct effects of FSH on bone. Amenorrheic women with a higher mean serum FSH ( $\sim 35 \mathrm{IU} / \mathrm{L})$ have greater bone loss than those with lower levels $(\sim 8 \mathrm{IU} / \mathrm{L})$ in the face of near-equal estrogen levels [11]. Likewise, in a recent study, patients with functional hypothalamic amenorrhea, in whom both FSH and estrogen were low, showed slight to moderate skeletal defects [12]. Furthermore, women harboring an activating FSH receptor (FSHR) polymorphism, rs6166, have lower bone mass and higher resorption markers [13]; this attests to a role for FSHRs in human physiology, and perhaps, even human pathophysiology. Consistent with these human studies, exogenously administration of FSH to rats augmented ovariectomy-induced bone loss [14, 15]. Moreover, an FSH antagonist reduced bone loss post-ovariectomy, as well as that induced by exogenous FSH [14, 15].

Clinical correlations between bone loss and serum FSH levels have been documented extensively. Most impressive is the Study of Women's Health across the Nations (SWAN), a longitudinal cohort of 2,375 peri-menopausal women. Not only was there a strong correlation between serum FSH levels and markers of bone resorption, a change in FSH levels over 4 years predicted decrements in bone 
Fig. 1 Bone loss at the most rapid rate in obese (top panel) or non-obese (bottom panel) begins 2-3 years before the last menstrual period (a), during which time serum estrogen levels are stable while serum FSH is rising (b). Adapted, with permission, from refs [3] and [6]
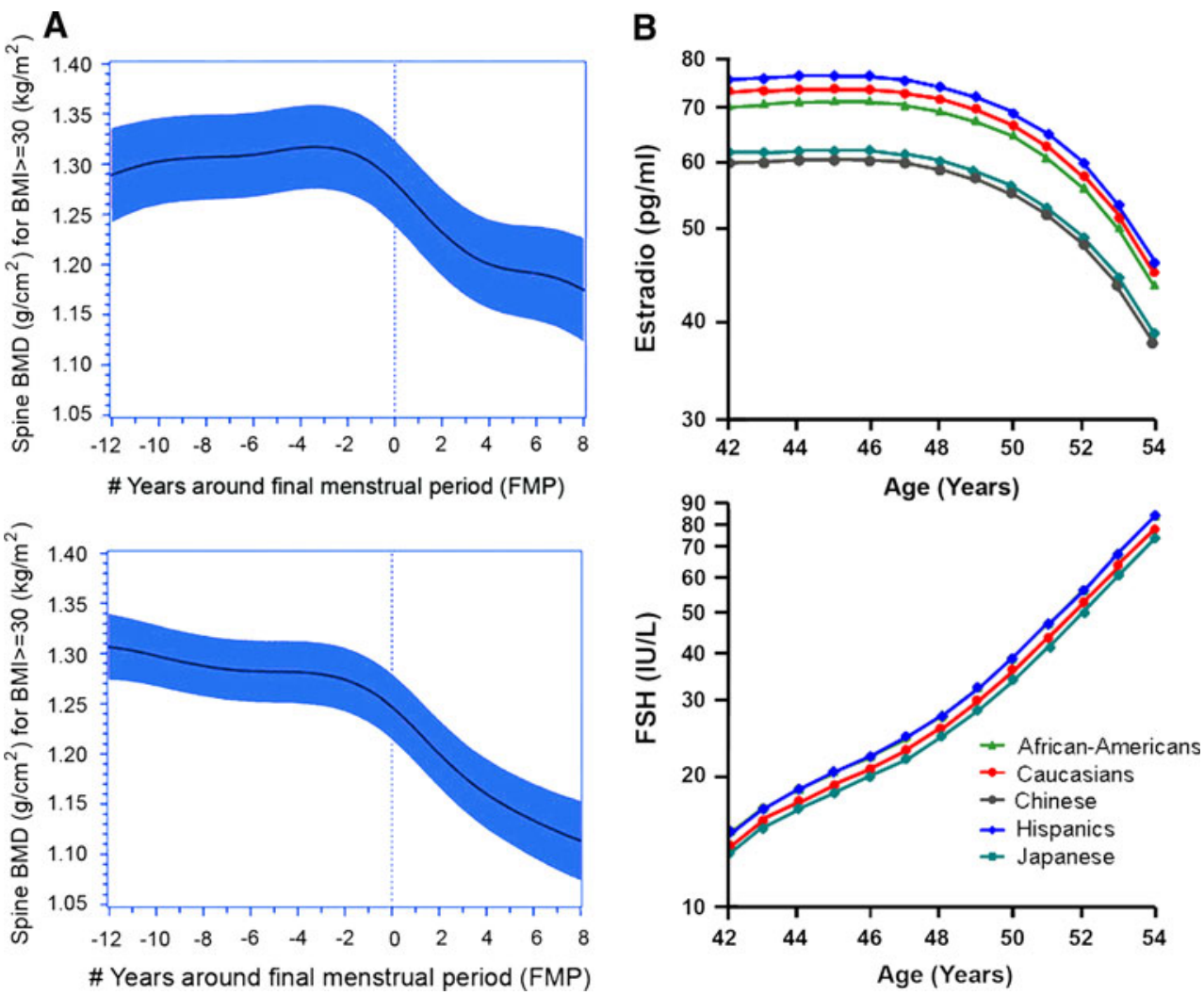

mass [16]. Analyses of data from Chinese women showed similar trends: a significant association between bone loss and high serum FSH [17, 18]. In a group of southern Chinese women aged between 45 and 55 years, those in the highest quartile of serum FSH lost bone at a 1.3- to 2.3-fold higher rate than those in the lowest quartile [19]. Likewise, a further detailed examination of the Third National Health Examination and Nutrition Survey (NHANES III) cohort of women between the ages of 42 and 60 years showed a strong correlation between serum FSH and femoral neck bone mineral density (BMD) [20]. A recent cross-sectional analysis of 92 postmenopausal women found that serum osteocalcin and C-terminal telopeptide of type I collagen (CTX) were both positively correlated with FSH, but not with estradiol [21]. Serum CTX was highest in the highest quartile of FSH [21]. Finally, the BONTURNO study group showed that women considered peri-menopausal based on their serum FSH levels of $>30 \mathrm{IU} / \mathrm{mL}$ had significantly higher bone turnover markers than age-matched women, despite having normal menses [22]. Together, these compelling studies prompt the use of FSH at least as a critical serum marker for identifying "fast bone losers" during the early phases of the menopausal transition [23].

In contrast, Gourlay et al. [24] fail to show a strong relationship between bone mass and FSH or indeed estrogen. This is surprising; such differences are likely related to distinct patient cohorts and statistical designs.
Interestingly, the same authors show an independent correlation between FSH and lean mass [25]. This association makes biological sense inasmuch as FSHRs are present on mesenchymal stem cells [9], which are known to have the propensity for adipocytic differentiation. However, studies have yet to determine whether FSH inhibits adipogenesis.

Mechanistically, others and we have shown that FSH increases osteoclast formation, function, and survival through a distinct FSHR isoform [9, 26-28]. Wu et al. [28] further showed that the osteoclastogenic response to FSH was abolished in mice lacking ITAM adapter signaling molecules. This suggested an interaction between FSH and immune receptor complexes, although the significance of this finding remains unclear. In a separate study, FSHR activation was shown also to enhance expression of receptor activator for NF- $\kappa \mathrm{B}$ (RANK) [29].

In addition to having direct effects on the osteoclast, FSH also indirectly stimulates osteoclast formation by releasing osteoclastogenic cytokines [30, 31]. It induces pre-osteoclasts to secrete IL- $1 \beta$, TNF- $\alpha$, and IL-6 in proportion to the surface expression of FSHRs [31]. In a study of 36 women between the ages of 20 and 50, serum FSH concentrations correlated with circulating cytokine concentrations [31, 32].

Two groups have, however, failed to identify FSHRs on osteoclasts, having likely used primers designed to detect the ovarian isoform $[33,34]$. We suggest that they look 
Fig. 2 Follicle-stimulating hormone contributes to skeletal regulation. Bone remodeling is under extensive and varied control mechanisms allowing integration and adaptation to a variety of stimuli. Rapid neural signals from the brain regulate osteoblastic control of the hematopoietic stem cell niche (labeled frequency modulation) as well as bone formation. Broad and relatively slower shifts in bone turnover are controlled by the pituitary hormones follicle-stimulating hormone (FSH) and thyroidstimulating hormone (TSH) largely targeting osteoclasts, and by members of the growth hormone $(\mathrm{GH})$ pathway targeting osteoblasts [38]

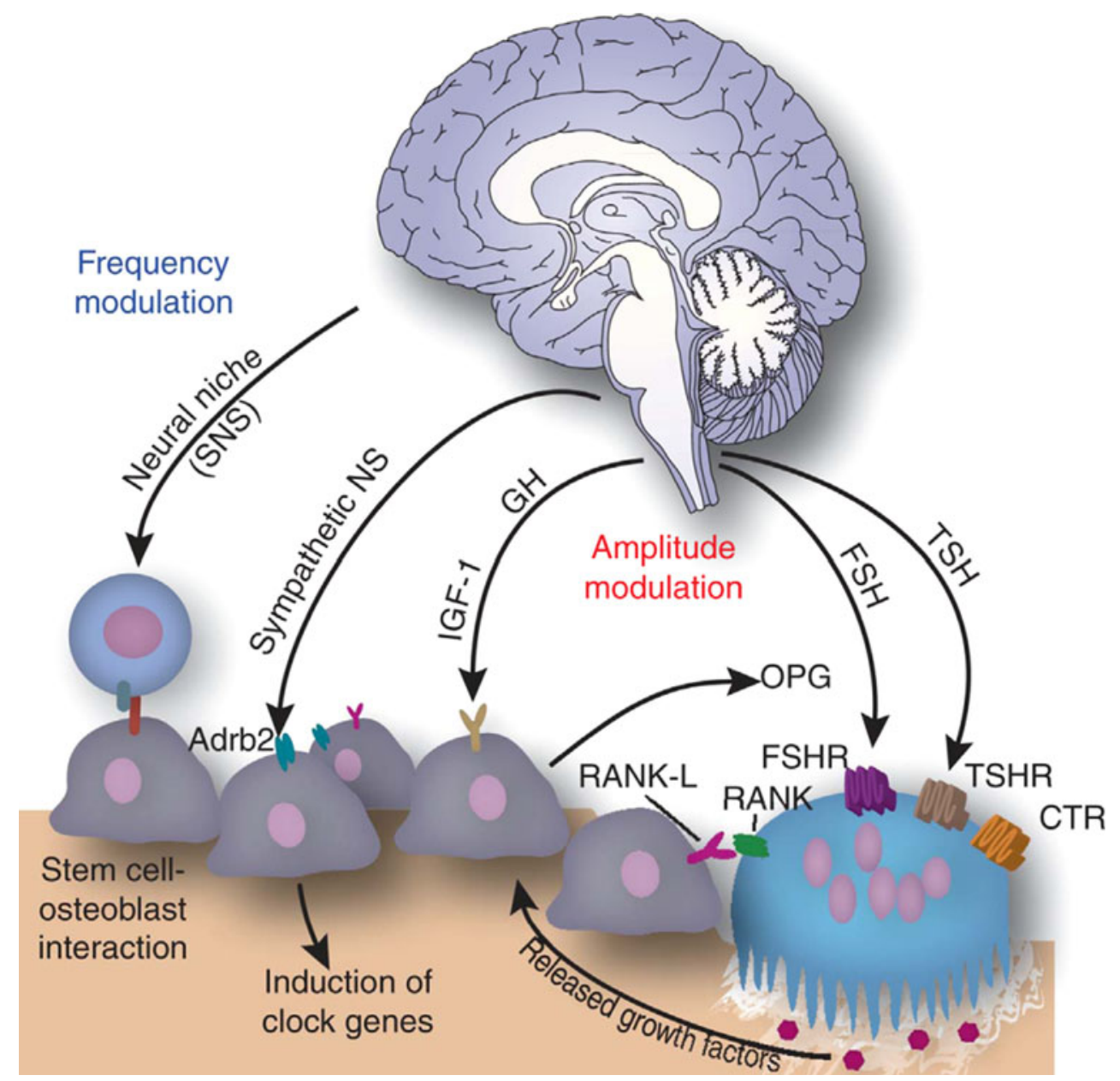

more closely using appropriate primers [26]. Furthermore, injection of FSH into mice with intact ovaries [34], or its transgenic overexpression [33], even in hpg mice, is unlikely to reveal pro-resorptive actions of FSH. This is because direct effects of FSH on the osteoclast will invariably be masked by the anti-resorptive and anabolic actions of the ovarian estrogen so released in response to FSH. It is admittedly difficult, therefore, to tease out the action of FSH from that of estrogen in vivo as FSH releases estrogen and their actions on the osteoclast are opposed. These negative studies therefore do not rule out direct receptor-mediated actions of FSH on the skeleton and skeletal cells demonstrated in both rodents and humans.

Whether lowering FSH in a hypogonadal state to prevent bone loss can be leveraged therapeutically remains to be determined. There is evidence that women with low FSH levels undergo less bone loss [11], and that the effectiveness of estrogen therapy is related to the degree of FSH suppression [35]. With that said, patients with pituitary hypogonadism can, in circumstances, lose bone. This was in fact elegantly demonstrated in an intervention study, wherein luperide treatment, and hence the lowering of FSH, did not prevent hypogonadal bone loss [36].
While this study proved that low estrogen is a cause of acute hypogonadal bone loss, this did not exclude a role for FSH in human skeletal homeostasis [36]. Specifically, the study did not examine the confounding effects of perturbations in GnRH, LH or indeed, inhibin; the latter stimulates bone formation [37]. We believe that rather than blocking FSH in acute hypogonadism, where the effect of low estrogen is likely to be overwhelming, FSH inhibition during the late peri-menopause, particularly when estrogen levels are normal and FSH is high, could potentially be of therapeutic significance. A highly selective approach, such as the use of a blocking antibody, is thus envisaged.

Acknowledgments M.Z., HCB and S.L. are supported by grants from the National Institutes of Health. J.I acknowledges the support of the American Federation for Aging Research. AZ is supported by the Italian Space Agency.

Disclosures M.Z. consults for Questcor and Amgen. M.Z. is also a named inventor of a pending patent application related to osteoclastic bone resorption and FSH filed by the Mount Sinai School of Medicine (MSSM). In the event the pending or issued patent is licensed, he would be entitled to a share of any proceeds MSSM receives from the licensee. J.I, H.C.B, A.Z, and S.L have nothing to disclose. 


\section{References}

1. E.C. Reifenstein Jr., F. Albright, The metabolic effects of steroid hormones in osteoporosis. J. Clin. Invest. 26, 24-56 (1947)

2. J. Iqbal, M. Zaidi, Understanding estrogen action during menopause. Endocrinology 150, 3443-3445 (2009)

3. M.R. Sowers, H. Zheng, M.L. Jannausch, D. McConnell, B. Nan, S. Harlow, J.F. Randolph Jr., Amount of bone loss in relation to time around the final menstrual period and follicle-stimulating hormone staging of the transmenopause. J. Clin. Endocrinol. Metabol. 95, 2155-2162 (2010)

4. M.R. Sowers, H. Zheng, D. McConnell, B. Nan, S. Harlow, J.F. Randolph Jr, Follicle-stimulating hormone and its rate of change in defining menopausal transition changes. J. Clin. Endocrinol. Metabol. 93, 3958-3964 (2008)

5. M.R. Sowers, M. Jannausch, D. McConnell, R. Little, G.A. Greendale, J.S. Finkelstein, R.M. Neer, J. Johnston, B. Ettinger, Hormone predictors of bone mineral density changes during the menopausal transition. J. Clin. Endocrinol. Metab. 91, 1261-1267 (2006)

6. J.F. Randolph Jr, M. Sowers, I.V. Bondarenko, S.D. Harlow, J.L. Luborsky, R.J. Little, Change in estradiol and follicle-stimulating hormone across the early menopausal transition: effects of ethnicity and age. J. Clin. Endocrinol. Metab. 89, 1555-1561 (2004)

7. G. Rannevik, S. Jeppsson, O. Johnell, B. Bjerre, Y. LaurellBorulf, L. Svanberg, A longitudinal study of the peri-menopausal transition: altered profiles of steroid and pituitary hormones. SHBG and bone mineral density. Maturitas 61, 67-77 (2008)

8. E. Abe, R.C. Marians, W. Yu, X.B. Wu, T. Ando, Y. Li, J. Iqbal, L. Eldeiry, G. Rajendren, H.C. Blair, T.F. Davies, M. Zaidi, TSH is a negative regulator of skeletal remodeling. Cell 115, 151-162 (2003)

9. L. Sun, Y. Peng, A.C. Sharrow, J. Iqbal, Z. Zhang, D.J. Papachristou, S. Zaidi, L.L. Zhu, B.B. Yaroslavskiy, H. Zhou, A. Zallone, M.R. Sairam, T.R. Kumar, W. Bo, J. Braun, L. CardosoLanda, M.B. Schaffler, B.S. Moonga, H.C. Blair, M. Zaidi, FSH directly regulates bone mass. Cell 125, 247-260 (2006)

10. M. Zaidi, Skeletal remodeling in health and disease. Nature Med. 13, 791-801 (2007)

11. B. Devleta, B. Adem, S. Senada, Hypergonadotropic amenorrhea and bone density: new approach to an old problem. J. Bone Miner. Metab. 22, 360-364 (2004)

12. A. Podfigurna-Stopa, P. Pludowski, M. Jarowski, R. Lorenc, A.R. Genazzani, B. Meczekalski, Skeletal status and body composition in young women with functional hypothalamic amenhorrea. Gynaecol. Endocrinol. (2011, Epub ahead of print)

13. D. Rendina, F. Gianfrancesco, G. De Filippo, D. Merlotti, T. Esposito, A. Mingione, R. Nuti, P. Strazzullo, G. Mossetti, L. Gennari, FSHR gene polymorphisms influence bone mineral density and bone turnover in post-menopausal women. Eur. J. Endocrinol. 163, 165-172 (2010)

14. S. Liu, Y. Cheng, M. Fan, D. Chen, Z. Bian, FSH aggravates periodontitis-related bone loss in ovariectomized rats. J. Dent. Res. 89, 366-371 (2010)

15. S. Liu, Y. Cheng, W. Xu, Z. Bian, Protective effects of folliclestimulating hormone inhibitor on alveolar bone loss resulting from experimental periapical lesions in ovariectomized rats. J. Endod. 36, 658-663 (2010)

16. M.R. Sowers, G.A. Greendale, I. Bondarenko, J.S. Finkelstein, J.A. Cauley, R.M. Neer, B. Ettinger, Endogenous hormones and bone turnover markers in pre- and perimenopausal women: SWAN. Osteoporos. Int. 14, 191-197 (2003)

17. Z.R. Xu, A.H. Wang, X.P. Wu, H. Zhang, Z.F. Sheng, X.Y. Wu, H. Xie, X.H. Luo, E.Y. Liao, Relationship of age-related concentrations of serum FSH and LH with bone mineral density, prevalence of osteoporosis in native Chinese women. Clin. Chim. Acta 400, 8-13 (2009)

18. X.Y. Wu, X.P. Wu, H. Xie, H. Zhang, Y.Q. Peng, L.Q. Yuan, X. Su, X.H. Luo, E.Y. Liao, Age-related changes in biochemical markers of bone turnover and gonadotropin levels and their relationship among Chinese adult women. Osteoporos. Int. 21, 275-285 (2009)

19. E. Cheung, S. Tsang, C. Bow, S. Yueng, C. Loomg, C.L. Cheung, A. Kan, S. Lo, S. Tam, G. Tang, A. Kung, Bone loss during menopausal transition in Chinese women. Maturitas 69, 50-56 (2011)

20. C.M. Gallagher, B.S. Moonga, J.S. Kovach, Cadmium, folliclestimulating hormone, and effects on bone in women age 42-60 years: NHANES III. Environ. Res. 110, 105-111 (2010)

21. A. Garcia-Martin, R. Reyes-Garcia, J.M. Garcia-Castro, P. RozasMoreno, F. Escobar-Jimenez, M. Munoz-Torres, Role of serum FSH measurement on bone resorption in postmenopausal women. Endocrine (2011, Epub ahead of print)

22. S. Adami, G. Bianchi, M.L. Brandi, S. Giannini, S. Ortolani, O. DiMunno, B. Frediani, M. Rossini, BONTURNO study group, determinants of bone turnover markers in healthy premenopausal women. Calcif. Tiss. Int. 82, 341-347 (2008)

23. M. Zaidi, C.H. Turner, E. Canalis, R. Pacifici, L. Sun, J. Iqbal, X.E. Guo, S. Silverman, S. Epstein, C.J. Rosen, Bone loss or lost bone: rationale and recommendations for the diagnosis and treatment of early postmenopausal bone loss. Curr. Osteoporos. Rep. 7, 118-126 (2009)

24. M.L. Gourlay, J.S. Preissner, C.A. Hammet-Stabler, J.B. Renner, J. Rubin, Follicle stimulating hormone and bioavailable estradiol are less important than weight and race in determining bone density in younger post-menopausal women. Osteoporos. Int. 22, 2699-2708 (2011)

25. M.L. Gourlay, B.L.Specker, C. Li, C.A. Hammett-Stabler, J.B. Renner, J.E. Rubin, Follicle-stimulating hormone is independently associated with lean mass but not BMD in younger postmenopausal women. Bone 50, 311-316 (2012)

26. L.J. Robinson, I. Tourkova, Y. Wang, A.C. Sharrow, M.S. Landau, B.B. Yaroslavskiy, L. Sun, M. Zaidi, H.C. Blair, FSH receptor isoforms and FSH-dependent gene transcription in human monocytes and osteocytes. Biochem. Biophys. Res. Commun. 394, 12-17 (2010)

27. L. Sun, Z. Zhang, L.L. Zhu, Y. Peng, X. Liu, J. Li, M. Agrawal, L.J. Robinson, J. Iqbal, H.C. Blair, M. Zaidi, Further evidence for a direct pro-resorptive actions of FSH. Biochem. Biophys. Res. Commun. 394, 6-11 (2010)

28. Y. Wu, J. Torchia, W. Yao, N.E. Lane, L.L. Lanier, M.C. Nakamura, M.B. Humphrey, Bone microenvironment specific roles of ITAM adapter signaling during bone remodeling induced by acute estrogen-deficiency. PLoS. One. 2, e586 (2007)

29. J.G. Cannon, B. Kraj, G. Sloan, Follicle-stimulating hormone promotes RANK expression on human monocytes. Cytokine 53, 141-144 (2011)

30. J. Iqbal, L. Sun, T.R. Kumar, H.C. Blair, M. Zaidi, Folliclestimulating hormone stimulates TNF production from immune cells to enhance osteoblast and osteoclast formation. Proc. Natl. Acad. Sci. USA 103, 14925-14930 (2006)

31. J.G. Cannon, M. Cortez-Cooper, E. Meaders, J. Stallings, S. Haddow, B. Kraj, G. Sloan, A. Mulloy, Follicle-stimulating hormone, interleukin-1, and bone density in adult women. Am. J. Physiol. Regul. Integr. Comp. Physiol. 298, R790-R798 (2010)

32. E.R. Gertz, N.E. Silverman, K.S. Wise, K.B. Hanson, D.L. Alekel, J.W. Stewart, C.D. Perry, S.N. Bhupathiraju, M.L. Kohut, M.D. Van Loan, Contribution of serum inflammatory markers to changes in bone mineral content and density in postmenopausal women: a 1-year investigation. J. Clin. Densitom. 13, 277-282 (2010) 
33. C.M. Allan, R. Kalak, C.R. Dunstan, K.J. McTavish, H. Zhou, D.J. Handlesman, M.J. Seibel, Follicle-stimulating hormone increases bone mass in female mice. Proc. Natl. Acad. Sci. USA. 107, 22629-22634 (2010)

34. V. Ritter, B. Thuering, P. Saint Mezard, N.H. Luong-Nguyen, Y. Seltenmeyer, U. Junker, B. Fournier, M. Susa, F. Morvan, Follicle stimulating hormone does not impact male bone mass in vivo or human male osteoclasts in vitro. Calcif. Tiss. Int. 82, 383-391 (2008)

35. H. Kawai, M. Furuhashi, N. Suganuma, Serum follicle stimulating hormone level is a predictor of bone mineral density in patients with hormone replacement therapy. Arch. Gynecol. Obstet. 269, 192-195 (2004)

36. M.T. Drake, L.K. McCready, K.A. Hoey, E.J. Atkinson, S. Khosla, Effects of suppression of follicle-stimulating hormone secretion on bone resorption markers in post-menopausal women. J. Clin. Endocrinol. Metabol. 95, 5063-5068 (2010)

37. K.M. Nicks, T.W. Fowler, D. Gaddy, Reproductive hormones and bone. Curr. Osteoporos. Rep. 8, 60-67 (2010)

38. M. Zaidi, Neural surveillance of skeletal homeostasis. Cell. Metabol. 1, 219-221 (2005) 